Article

\title{
Evaluation of the Bactericidal and Fungicidal Activities of Poly([2-(methacryloyloxy)ethyl]trimethyl Ammonium Chloride)(Poly (METAC))-Based Materials
}

\author{
Toshiki Shiga ${ }^{1}$, Hiromitsu Mori ${ }^{1}$, Keiichi Uemura ${ }^{2}$, Ryota Moriuchi ${ }^{3}$, Hideo Dohra ${ }^{3}$, \\ Aika Yamawaki-Ogata ${ }^{4}$, Yuji Narita ${ }^{4}$, Akihiro Saito ${ }^{1}$ and Yohei Kotsuchibashi ${ }^{1, *}$ \\ 1 Department of Materials and Life Science, Shizuoka Institute of Science and Technology, 2200-2 Toyosawa, \\ Fukuroi, Shizuoka 437-8555, Japan; giants_421_10@yahoo.co.jp (T.S.); 1822008.mh@sist.ac.jp (H.M.); \\ saito.akihiro@sist.ac.jp (A.S.) \\ 2 Chutoen-General Medical Center, 1-1 Shobugaike, Kakegawa, Shizuoka 436-8555, Japan; \\ k.uemura@fsinet.or.jp \\ 3 Research Institute of Green Science and Technology, Shizuoka University, 836 Ohya, Suruga-ku, \\ Shizuoka City, Shizuoka 422-8529, Japan; moriuchi.ryota@shizuoka.ac.jp (R.M.); \\ dora.hideo@shizuoka.ac.jp (H.D.) \\ 4 Department of Cardiac Surgery, Nagoya University Graduate School of Medicine, 65 Tsurumai-cho, \\ Showa-ku, Nagoya, Aichi 466-8550, Japan; aika@med.nagoya-u.ac.jp (A.Y.-O.); \\ ynarita@med.nagoya-u.ac.jp (Y.N.) \\ * Correspondence: kotsuchibashi.yohei@sist.ac.jp; Tel.: +81-538-45-0111
}

Received: 27 July 2018; Accepted: 21 August 2018; Published: 26 August 2018

\begin{abstract}
Poly([2-(methacryloyloxy)ethyl]trimethyl ammonium chloride) (METAC) and the gels were prepared and evaluated for their bactericidal and fungicidal activities. The antimicrobial properties of poly(METAC) were tested against Escherichia coli (E. coli), Bacillus subtilis (B. subtilis), Saccharomyces cerevisiae (Sa. cerevisiae), methicillin-susceptible Staphylococcus aureus (MSSA), methicillin-resistant Staphylococcus aureus (MRSA), Pseudomonas aeruginosa (P. aeruginosa), and Candida albicans (C. albicans). Moreover, the structural forms of the linear and cross-linked poly(METAC) were investigated for their influences on bacterial aggregation, precipitation, and cell-death. To our knowledge, this is the first report on the comparison of the antimicrobial properties of poly(METAC) and poly(METAC)-gels. The bactericidal and fungicidal activities were evaluated by determining minimum inhibitory concentrations (MICs), UV-Vis spectroscopy, and fluorescence and confocal microscopies. The MICs were found to be 123 (MSSA), 123 (MRSA), 123 (P. aeruginosa), 370 (E. coli), 123 (B. subtilis), 370 (C. albicans), and $370 \mu \mathrm{g} / \mathrm{mL}$ (Sa. cerevisiae), as determined by broth dilution, and 370 (MSSA), 370 (MRSA), 370 (P. aeruginosa), 3300 (E. coli), 370 (B. subtilis), 1100 (C. albicans), and $>10,000 \mu \mathrm{g} / \mathrm{mL}$ (Sa. cerevisiae), as determined by paper disc diffusion (on solid medium). The poly(METAC)-gels achieved rapid adsorption/precipitation of bacteria via the cationic surface charge. Thus, these poly(METAC)-based polymers can potentially be used as antibacterial materials.
\end{abstract}

Keywords: antimicrobial polymer; cationic polymer; poly([2-(methacryloyloxy)ethyl]trimethyl ammonium chloride); drug-resistant bacteria

\section{Introduction}

Antimicrobial polymers have been receiving substantial attention owing to their multiple advantages, such as long-term activity, limited residual toxicity, chemical stability, non-volatility, and non-penetration via skin [1]. Cationic polymers are one of the most studied antimicrobial polymers $[2,3]$. The accepted mechanism by which cationic polymers lead to bacterial death is as 
follows: 1 . adsorption onto the bacterial cell surface, 2 . diffusion through the cell wall, 3 . binding to the cytoplasmic membrane, and 4. disruption of the bacterial membrane [4]. This mechanism is effective regardless of the types of bacteria. Therefore, it is expected that the cationic polymers can also show an antimicrobial effect against drug-resistant bacteria. Recently, the World Health Organization (WHO) has listed the drug-resistant bacteria that could shortly become a menace to humans [5]. For example, in the US, the methicillin-resistant Staphylococcus aureus (MRSA) causes more deaths than human immunodeficiency virus (HIV) [6]. Very recently, Su et al. prepared cationic polypeptide-based polymers that present antimicrobial properties when administered in MRSA-infected mice $[7,8]$. The poly(hexamethylene biguanide), another type of cationic polymer, is approved by the Food and Drug Administration (FDA), and there are no reports on emergent drug-resistant bacteria $[9,10]$. Poly([2-(methacryloyloxy)ethyl]trimethylammonium chloride) (poly(METAC)) is one of the methacrylate types of cationic polymer, consisting of quaternary ammonium cations. The methacrylate monomer can be polymerized by (living/controlled) radical polymerizations and can also be copolymerized with other types of typical (meth)acrylate monomers [11-13]. The development of polymeric synthesis has facilitated access to functional polymers, allowing not only the control of molecular weights and molecular weight distribution, but also the control of the physicochemical properties of the polymeric structures $[14,15]$. Stopiglia et al. showed that the METAC monomer presents antimicrobial properties against 31 kinds of Candida albicans (C. albicans) [16]. Prijick et al. succeeded in preventing the formation of a C. albicans biofilm on a surface coated with copolymers of poly(METAC) [17]. The poly(METAC) has also been conjugated with natural polymers. The cotton grafting poly(METAC) shows antimicrobial properties against Gram-positive and Gram-negative bacteria, and this effect increases with the amount of grafted poly(METAC) [18]. A wool-modified surface was combined with the chitosan grafting poly(METAC), and the conjugated materials shows efficient antimicrobial properties [19]. In this study, the antimicrobial properties of poly(METAC) were tested against Escherichia coli (E. coli), Bacillus subtilis (B. subtilis), Saccharomyces cerevisiae (Sa. cerevisiae), methicillin-susceptible Staphylococcus aureus (MSSA), MRSA, Pseudomonas aeruginosa (P. aeruginosa), and C. albicans. In addition, poly(METAC)-gel was prepared to allow a comparison of the two different structural forms, linear and cross-linked poly(METAC), on their ability to induce bacterial aggregation, precipitation, and cell-death. To our knowledge, this is the first report on a comparison of antimicrobial properties of poly(METAC) and poly(METAC)-gel (Figure 1). The minimum inhibitory concentration (MIC) was determined by broth dilution and paper disc diffusion methods, and the bacterial aggregation, precipitation, and cell-death were measured by UV-Vis spectroscopy, and fluorescence and confocal microscopies.
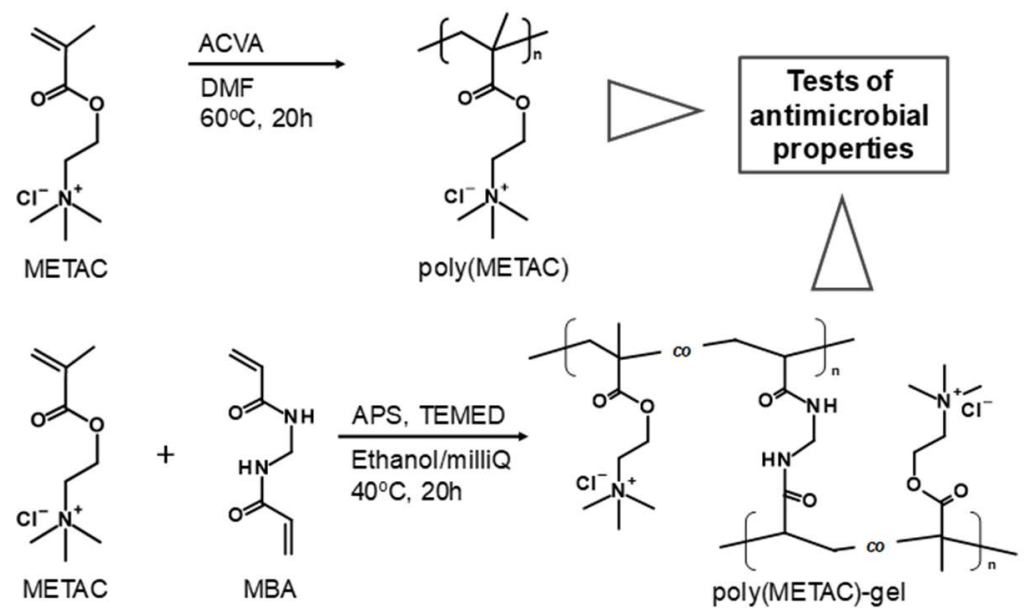

Figure 1. Synthesis of poly(METAC) and poly(METAC)-gel polymerized by a free radical polymerization. 


\section{Materials and Methods}

\subsection{Materials}

[2-(methacryloyloxy)ethyl]trimethylammonium chloride (METAC) (80 wt \% in water), 4, $4^{\prime}$-azobis(4-cyanovaleric acid) (ACVA), and $N, N^{\prime}$-methylenebis(acrylamide) (MBA) were purchased from Sigma-Aldrich (St Louis, MO, USA) and were used as received. All other chemicals and solvents were used as received. Escherichia coli JM109 [20], Bacillus subtillis 168, and a strain of Saccharomyces cerevisiae isolated from commercially obtained dry yeast were (Nisshin Seifun Group Inc., Tokyo, Japan) used as representatives for Gram-negative, Gram-positive bacteria, and fungi B. subtilis 168 (JCM 10629) was obtained from the Japan Collection of Microorganisms (JCM, Ibaraki, Japan), BioResource Research Center, RIKEN, Ibaraki, Japan. Pseudomonas aeruginosa NBRC $12689^{\mathrm{T}}$ (=ATCC 10145) and Candida albicans NBRC $1385^{\mathrm{T}}$ (=ATCC 18804) were supplied from the Biological Resource Center, NITE (NBRC), Tokyo, Japan. Methicillin-susceptible strain KUSA01 and a resistant strain KUSA02 of Staphylococcus aureus used in this study were clinically isolated.

\subsection{Preparation of Poly(METAC) and Poly(METAC)-gel}

Free radical polymerization was employed to synthesize poly(METAC) and poly(METAC)-gel. Poly(METAC) was prepared as follows. METAC (2.60 g (including water), $10 \mathrm{mmol}$ ) and ACVA (14.0 mg, $0.050 \mathrm{mmol})$ ([METAC $\left.]_{0} /[\mathrm{ACVA}]_{0}=200 / 1\right)$ were dissolved in $5 \mathrm{~mL}$ of $\mathrm{N}, \mathrm{N}$-dimethylformamide (DMF). After degassing with nitrogen gas for $30 \mathrm{~min}$, polymerization was allowed for $24 \mathrm{~h}$ at $60{ }^{\circ} \mathrm{C}$. The resulting poly(METAC) was purified by dialysis, firstly against ethanol/water $1 / 1(v / v)$ and then water, and dried by lyophilization. Poly(METAC)-gel was prepared using different amounts of a cross-linker of $N, N^{\prime}$-methylenebis(acrylamide) (MBA). METAC (2.60 g (including water), $10 \mathrm{mmol}$ ) and MBA (10, 20, 30, or $50 \mathrm{mg}$; 0.065, 0.13, 0.19, or $0.32 \mathrm{mmol})$ were dissolved in $10 \mathrm{~mL}$ of a mixture solution of ethanol/water $1 / 1(v / v)$ at $40{ }^{\circ} \mathrm{C}$. Two-hundred microliters of ammonium peroxodisulfate (APS) solution (10 wt \% in water) and $20 \mu \mathrm{L}$ of $N, N, N^{\prime}, N^{\prime}$-Tetramethylethylenediamine (TEMED) were added to the mixture solution, and this was incubated for $20 \mathrm{~h}$ at $40{ }^{\circ} \mathrm{C}$, to allow polymerization. The resulting poly(METAC)-gel was purified via immersion, firstly in ethanol/water $1 / 1(v / v)$, and then in ethanol. After the purification, the poly(METAC)-gel was dried under reduced pressure. Poly(METAC)-gels were labeled as the poly(METAC)-gel- $X$, in which $X$ is the mol \% of cross-linker in the total of METAC monomer and cross-linker (i.e., $X=0.65,1.28,1.86$, and 3.10). For example, the 0.65 was calculated via the $(0.065 /(10+0.065)) \times 100$.

\subsection{Determination of Minimum Inhibitory Concentration (MIC) of Poly(METAC)}

MICs were determined by broth dilution and paper disc diffusion methods. Luria broth was used for E. coli and B. subtillis, Müller Hinton broth (MHB) for S. aureus, cation-adjusted MHB for P. aeruginosa, potato-dextrose broth for S. cerevisiae, and MHB containing glucose $(2 \%(w / v))$ and methylene blue $(0.5 \mu \mathrm{g} / \mathrm{mg})$ for C. albicans. Except for P. aeruginosa, corresponding agar media were used for the paper disc diffusion method. Müller Hinton agar medium was used for P. aeruginosa. The suitable broths for S. aureus, P. aeruginosa, and C. albicans were selected using the performance standards for antimicrobial susceptibility testing of the medical field. Other bacteria were incubated using the typical broths. Bacterial suspensions were prepared via the turbidity (McFarland standards) in accordance with each bacterial performance standards. For the broth dilution method, microbial cells $\left(150 \mu \mathrm{L}\right.$ of the overnight culture fluid) cultivated overnight at $37^{\circ} \mathrm{C}$ were inoculated into fresh liquid medium supplemented with poly(METAC) and subsequently incubated for $24 \mathrm{~h}$ at $37^{\circ} \mathrm{C}$ with shaking at $150 \mathrm{rpm}$. MIC was defined as the minimum poly(METAC) concentration that completely inhibited microbial growth. For the paper disc diffusion method, microbial cells were spread onto agar medium, over which a series of discs containing $0-1.0 \%(w / v)$ poly(METAC) were placed. Cells were 
cultivated for $24 \mathrm{~h}$ at $37^{\circ} \mathrm{C}$ and growth of microorganisms around the discs were observed. MIC was defined as the minimum poly(METAC) concentration in discs leading to growth inhibition zones.

\subsection{Evaluation of Bacterial Aggregation/Precipitation by Poly(METAC) and Poly(METAC)-gel}

B. subtilis cells grown in LB liquid medium until the mid-exponential phase were exposed to poly(METAC) or poly(METAC)-gel-1.28 at different concentrations (1-5 mg/1.5 mL) for $24 \mathrm{~h}$. The appropriate amounts of poly(METAC) or poly(METAC)-gel-1.28) were dissolved/dispersed in $500 \mu \mathrm{L}$ milliQ and mixed with B. subtilis suspension $(1 \mathrm{~mL})$. After $24 \mathrm{~h}$, the transmittance of the supernatants was measured using UV-Vis spectroscopy at $500 \mathrm{~nm}$.

\subsection{Evaluation of the Bactericidal Effect of Poly(METAC) and Poly(METAC)-gel}

E. coli cells grown in LB liquid medium until the mid-exponential phase were exposed to poly(METAC) or MilliQ water (as control) and immediately subjected for the next procedures. To evaluate the bactericidal activity of the polymer, cells treated with poly(METAC) were stained with SYTO9 and propidium iodide (PI) using the LIVE/DEAD BacLight Bacterial Viability Kit (Life Technologies, Carlsbad, CA, USA), by following the manufacturer's instructions, and observed under a fluorescence microscope. To evaluate bacterial survival in the presence of poly(METAC), E. coli cells exposed to different concentrations of poly(METAC) were washed once with phosphate buffered saline [21], spread on LB agar medium, and incubated at $37^{\circ} \mathrm{C}$ for $24 \mathrm{~h}$, after which the colonies were counted. Adsorbed bacteria on poly(METAC)-gel were observed by confocal microscopy. The poly(METAC)-gels were immersed into an E. coli suspension for $10 \mathrm{~min}$ and washed 2 times with large amounts of $\mathrm{pH} 7.4$ phosphate-buffered saline (PBS). Bacterial cells on the poly(METAC)-gels were stained using the LIVE/DEAD BacLight Bacterial Viability Kit.

\subsection{Characterizations}

Number-average molecular weight $\left(M_{\mathrm{n}}\right)$ and molecular weight distribution $\left(M_{\mathrm{w}} / M_{\mathrm{n}}, M_{\mathrm{w}}\right.$ : weight-average molecular weight) of the synthesized poly(METAC) were determined by gel permeation chromatography (GPC) (Shimadzu, Kyoto, Japan) at room temperature, with SB-802.5 HQ and SB-804 HQ columns (Shodex, Tokyo, Japan) connected to a RID-20A refractive index detector (Shimadzu, Kyoto, Japan). Elution was performed with $0.5 \mathrm{M}$ sodium acetate/0.5 M acetic acid buffer at a flow rate of $1.0 \mathrm{~mL} / \mathrm{min}$. Transmittance of mixture suspensions of bacteria and polymeric materials at $500 \mathrm{~nm}$ was recorded at $25{ }^{\circ} \mathrm{C}$ on a UV-Vis spectrometer V-650 (JASCO International Co., Ltd., Tokyo, Japan). A microplate reader Infinite M1000-SSY (TECAN, Kanagawa, Japan), a fluorescence microscope MX (MEIJI Techno, Saitama, Japan) with an excitation light source FL-PWJ (MEIJI Techno, Saitama, Japan), and a confocal microscope LSM 700 (Carl Zeiss, Oberkochen, Germany) were used to evaluate the bactericidal effect of poly(METAC) copolymers.

\section{Results}

Molecular weight $\left(M_{n}\right)$ and molecular weight distribution $\left(M_{\mathrm{w}} / M_{\mathrm{n}}\right)$ of poly(METAC) were determined to be $87,400 \mathrm{~g} / \mathrm{mol}$ and 2.83 , respectively (measured by GPC). The synthesized poly(METAC) inhibited the growth of all the tested microorganisms: Gram-positive bacteria (S. aureus and B. subtilis), Gram-negative bacteria (P. aeruginosa and E. coli), and yeasts (C. albicans and Ss. cerevisiae). The determined minimum inhibitory concentrations (MICs) for these microorganisms are shown in Table 1. The MICs determined by broth dilution method (in liquid media) were found to be $123 \mu \mathrm{g} / \mathrm{mL}$ (MSSA), $123 \mu \mathrm{g} / \mathrm{mL}$ (MRSA), $123 \mu \mathrm{g} / \mathrm{mL}$ (P. aeruginosa), $370 \mu \mathrm{g} / \mathrm{mL}$ (E. coli), $123 \mu \mathrm{g} / \mathrm{mL}$ (B. subtilis), $370 \mu \mathrm{g} / \mathrm{mL}$ (C. albicans), and $370 \mu \mathrm{g} / \mathrm{mL}$ (Sa. cerevisiae). The MICs determined by paper disc diffusion method (on solid media) were found to be $370 \mu \mathrm{g} / \mathrm{mL}$ (MSSA), $370 \mu \mathrm{g} / \mathrm{mL}$ (MRSA), $370 \mu \mathrm{g} / \mathrm{mL}$ (P. aeruginosa), $3300 \mu \mathrm{g} / \mathrm{mL}$ (E. coli), $370 \mu \mathrm{g} / \mathrm{mL}$ (B. subtilis), $1100 \mu \mathrm{g} / \mathrm{mL}$ (C. albicans), and $>10,000 \mu \mathrm{g} / \mathrm{mL}$ (Sa. cerevisiae). The MICs of broth dilution method were lower than that of paper disc diffusion method, for all the microorganisms. These results suggested that 
the poly(METAC) exhibits antibacterial properties against Gram-positive bacteria, Gram-negative bacteria, and yeasts. Moreover, it presents similar antibacterial levels against both of MSSA and MRSA. Rawlinson et al. reported resistance of $S$. aureus to a cationic polymer of poly(2-(dimethylamino ethyl)methacrylate)(poly(DMAEMA)), when compared with S. epidermidis. The resistance was explained as resulting from the low negative charge and hydrophobicity of the $S$. aureus surface [22]. In fact, the MICs of every S. aureus strain (clinical isolate species, two MSSA and six MRSA) were all over $3200 \mu \mathrm{g} / \mathrm{mL}$. On the other hand, the MICs of eleven types of S. epidermidis were in the range $25-100 \mu \mathrm{g} / \mathrm{mL}$. Moreover, the adhesion of poly(DMAEMA) to S. aureus was lower than that of S. epidermidis, as measured by flow cytometry. The polymeric structures also affect the antimicrobial and adsorptive properties on the target bacteria. Shirbin et al. prepared a macroporous cryogel using cationic polypeptide of poly(lysine)- $b$-poly(valine), and the cryogel showed a "trap and kill" effect against E. coli [23]. The cationic four-arm star glycopolymer-peptides showed different MICs depending on the glycol-types such as glucose, galactose, or mannose (E. coli: 10-256 $\mu \mathrm{g} / \mathrm{mL}$, P. aeruginosa: $32-512 \mu \mathrm{g} / \mathrm{mL}$, and S. aureus 16-128 $\mu \mathrm{g} / \mathrm{mL}$ ) [24].

Table 1. Minimum inhibitory concentrations (MICs) of poly(METAC) synthesized in this study.

\begin{tabular}{cccc}
\hline & \multicolumn{2}{c}{ MIC $(\mu \mathrm{g} / \mathrm{mL}) *$} \\
\cline { 2 - 4 } S. aureus & in Liquid Media & on Solid Media \\
\cline { 2 - 4 } & $\mathrm{MR}$ & 123 & 370 \\
\hline P. aeruginosa & 123 & 370 \\
E. coli & 123 & 370 \\
B. subtilis & 370 & 3300 \\
C. albicans & 123 & 370 \\
Sa. cerevisiae & 370 & 1100 \\
\hline
\end{tabular}

* MICs were determined by broth dilution method (in liquid media) and paper disc diffusion method (on solid media). Please see Materials and Methods for detailed experimental conditions. S. aureus, Staphylococcus aureus; P. aeruginosa, Pseudomonas aeruginosa; E. coli, Escherichia coli; B. subtilis, Bacillus subtilis; C. albicans, Candida albicans; Sa. cerevisiae, Saccharomyces cerevisiae; MS, methicillin-susceptible strain; MR, methicillin-resistant strain.

Next, poly(METAC)-gels were tested on their abilities to induce bacterial aggregation, precipitation, and cell-death. The poly(METAC)-gels-1.28 were prepared via redox polymerization using $N, N^{\prime}$-methylenebis(acrylamide) as a cross-linker [25]. Figure 2 shows the transmittance changes of $B$. subtilis suspensions treated by poly(METAC) or poly(METAC)-gel-1.28 after $24 \mathrm{~h}$. The control showed that less than $5 \%$ of the observed transmittances were due to the suspended B. subtilis. Similarly, less than $5 \%$ of the observed transmittances of the B. subtilis suspensions were due to the adding of the poly(METAC) solution, which were similar to control. On the other hand, the transmittance tended to increase with the amount of poly(METAC)-gel-1.28, and reached $90 \%$ with $2 \mathrm{mg}$ of the gel. The weak bacterial aggregation/precipitation in the presence of poly(METAC) is caused by the electrostatic repulsion of the adsorbing poly(METAC) on the bacterial surface. The bacteria trapped on the poly(METAC)-gel-1.28 cannot be re-suspended via the electrostatic interaction between the anionic bacterial surface and cationic gel surface, resulting in the high transmittance values. In the nonionic poly(2-hydroxyethyl methacrylate (HEMA))-gel, the transmittance was lower than $5 \%$ (Figure S1). The interaction of nonionic poly(HEMA)-gels with bacteria was smaller than that of the cationic polymers. Similar results were reported by Berlutti's group [26]. Williams et al. prepared poly(METAC)-based cationic temperature-responsive gels, which show bacteriostatic and bactericidal properties against $S$. aureus when compared with that of nonionic gels [27]. 


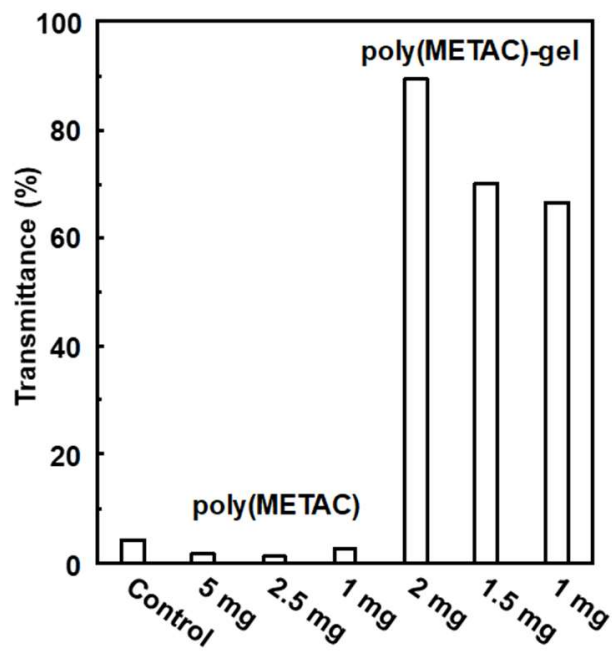

Figure 2. Transmittance measurement of the suspensions of poly(METAC) or poly(METAC)-gel-1.28 with B. subtilis. The B. subtilis suspensions were exposed to poly(METAC) or poly(METAC)-gel-1.28 at different concentrations for $24 \mathrm{~h}$. The total amount of solution was $1.5 \mathrm{~mL}$. After $24 \mathrm{~h}$, the transmittances of supernatants were measured at $500 \mathrm{~nm}$.

Next, the biocidal ability of the poly(METAC) and poly(METAC)-gels was investigated. Figure 3 shows the LIVE/DEAD test of E. coli in the presence of MilliQ (Figure 3A) or $1 \mathrm{wt} \%$ of poly(METAC) (Figure 3B). The E. coli were stained with SYTO9/PI, which leads to the exhibition of red fluorescence by the death cells due to the treatment with poly(METAC). In another experiment, E. coli were exposed to poly(METAC) at different concentrations $(10 \mathrm{mg} / \mathrm{mL}-41 \mu \mathrm{g} / \mathrm{mL})$, spread on the nutrient agar, and the colony forming units (CFUs) were measured after $24 \mathrm{~h}$. Figure $3 \mathrm{C}$ shows the $\mathrm{CFU}$ at each poly(METAC) concentration. CFUs decreased with increasing poly(METAC) concentration, and reached a plateau (about $100 \mathrm{CFU} / \mathrm{mL}$ ) at over $1.1 \mathrm{mg} / \mathrm{mL}$. The prevention of the E. coli growth by poly(METAC) was about 0.4 million-fold higher than that of the control without poly(METAC). Adsorbed bacteria on poly(METAC)-gels were observed by the confocal microscopy. The poly(METAC)-gels were immersed in the E. coli suspension for $10 \mathrm{~min}$, and were washed 2 times with large amounts of $\mathrm{pH}$ 7.4 PBS. The bacteria on the poly(METAC)-gels were stained with the live/dead agent. Figure 4A-C shows confocal microscopy images of the poly(METAC)-gels with different cross-linker amounts, $0.65,1.86$, and $3.10 \mathrm{~mol} \%$. Almost all bacteria on the poly(METAC)-gels were dead as shown by the red fluorescence. The adsorbed bacteria were also observed by scanning electron microscopy (SEM) measurement (Figure 4D). He et al. prepared a cationic hydrogel film via a light-triggered cross-linking [28]. The antimicrobial activity against E. coli was shown to depend on the amount of the ethylene glycol-based cross-linkers. At $20 \%$ of cross-linking degree, approximately $100 \%$ of antibacterial activity was reached. Yang et al. prepared a hydrogel consisting of METAC and nonionic monomers bound by disulfide bonds, and the gels successfully prevented the adsorption of proteins and E. coli on the surface [29]. The physical destruction of the adsorbing E. coli on the gel surface was observed via SEM measurement. Other nonionic polymers such as ethylene glycol-based copolymers also can prevent the adsorption of bacteria [30,31]. The METAC is a methacrylate type of monomer and the polymeric structure can be easily controlled via a combination of living radical polymerizations and click chemistry. The antibacterial properties will be customized by the structural formulation of poly(METAC). 

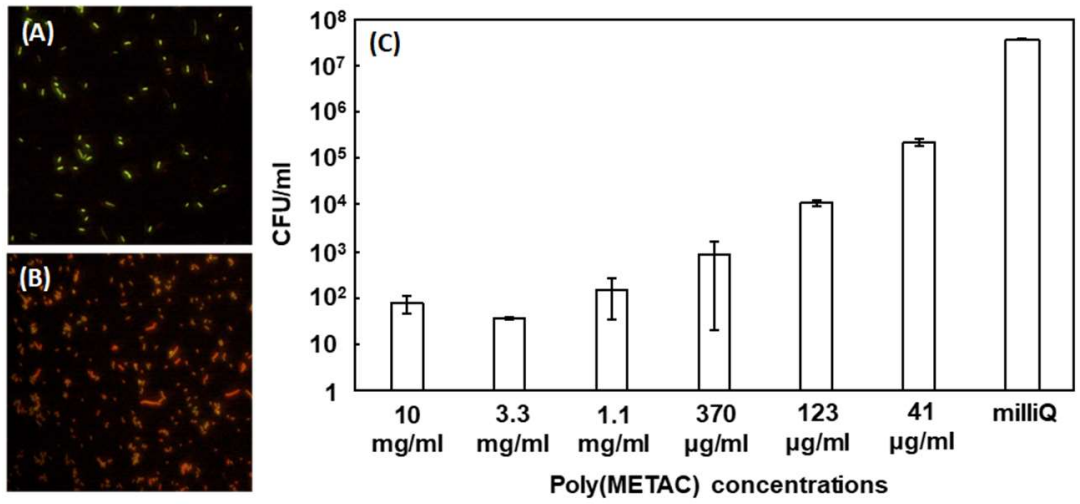

Figure 3. LIVE/DEAD test of E. coli in (A) milliQ and (B) $1 \mathrm{wt} \%$ of poly(METAC). The E. coli were stained with SYTO9 and PI. (C) The survival test of E. coli in the presence of poly(METAC) at different concentrations. The exposed E. coli by poly(METAC) were washed using phosphate-buffered saline (PBS) and were spread on medium to count the number of survived E. coli cells.
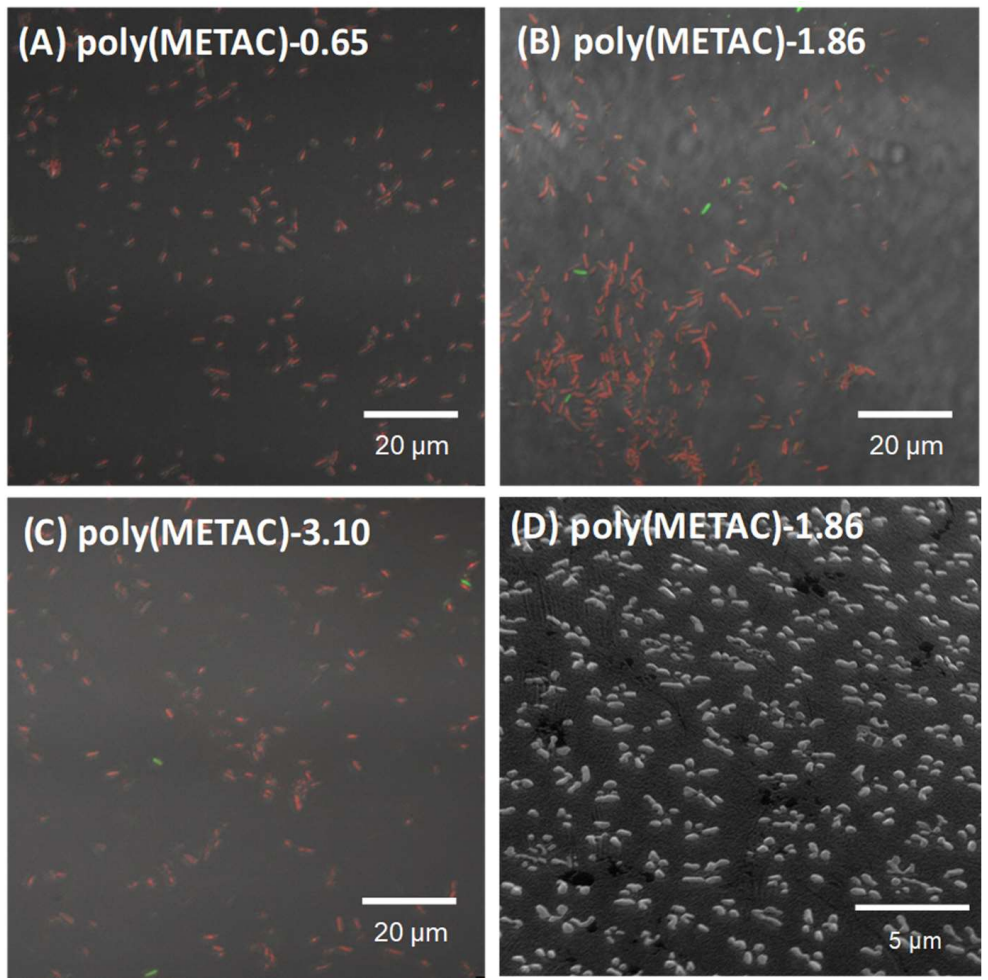

Figure 4. Confocal images of LIVE/DEAD test of E. coli on poly(METAC)-gels at various crosslinker amount; (A) $0.65 \mathrm{~mol} \%$, (B) $1.86 \mathrm{~mol} \%$, and (C) $3.10 \mathrm{~mol} \%$. The E. coli were stained with SYTO9 and PI. (D) Scanning electron microscope (SEM) images of the adsorbing E. coli on poly(METAC)-gels-1.86.

\section{Conclusions}

In conclusion, poly([2-(methacryloyloxy)ethyl]trimethyl ammonium chloride) (METAC) and poly(METAC)-gels were evaluated for their bactericidal and fungicidal activities. The MICs were found to be 123 (MSSA), 123 (MRSA), 123 (P. aeruginosa), 370 (E. coli), 123 (B. subtilis), 370 (C. albicans), and $370 \mu \mathrm{g} / \mathrm{mL}$ (Sa. cerevisiae) as determined by broth dilution. And, the MICs measured by paper disc diffusion method (on solid media) were 370 (MSSA), 370 (MRSA), 370 (P. aeruginosa), 3300 (E. coli), 370 (B. subtilis), 1100 (C. albicans), and $>10,000 \mu \mathrm{g} / \mathrm{mL}$ (Sa. cerevisiae). Importantly, poly(METAC) showed similar levels of antibacterial activity against both MSSA and MRSA. The poly(METAC)-gels 
showed rapid adsorption/precipitation of bacteria, with almost all bacteria being killed via the adsorption on poly(METAC)-gels within $10 \mathrm{~min}$. The METAC is a relatively low-cost monomer and can be copolymerized with typical (meth)acrylate and (meth)acrylamide monomers via the living radical polymerizations. Moreover, it is also easy to combine with the existing water-soluble polymers, such as poly(vinyl alcohol), poly(ethylene glycol), alginic acid, etc. The antibacterial properties will be customized by the structural formulation of poly(METAC), and the properties will be applied in the antibacterial dressing materials.

Thus, these poly(METAC)-based copolymers can potentially be used as antibacterial materials.

Supplementary Materials: The following are available online at http:/ /www.mdpi.com/2073-4360/10/9/947/s1, Figure S1: Transmittance measurement of the suspensions of poly(METAC)-gel with B. subtilis.

Author Contributions: Conceptualization, A.S. and Y.K.; methodology, K.U., R.M., H.D., A.Y.-O., Y.N., A.S. and Y.K.; formal analysis, T.S. and H.M.; investigation, all authors; writing, A.S. and Y.K.

Funding: This work was partially supported by research funds from Program for Building Regional Innovation Ecosystems and JSPS KAKENHI (16K16402).

Acknowledgments: We are grateful to K. Hayakawa and Y. Wakikawa of the Advanced Instrumental Analysis Center at the Shizuoka Institute of Science and Technology for their technical support.

Conflicts of Interest: The authors declare no conflicts of interest.

\section{References}

1. Santos, M.R.E.; Fonseca, A.C.; Mendonça, P.V.; Branco, R.; Serra, A.C.; Morais, P.V.; Coelho, J.F.J. Recent developments in antimicrobial polymers: A review. Materials 2016, 9, 599. [CrossRef] [PubMed]

2. Jain, A.; Duvvuri, L.S.; Farah, S.; Beyth, N.; Domb, A.J.; Khan, W. Antimicrobial polymers. Adv. Heal. Mater. 2014, 3, 1969-1985. [CrossRef] [PubMed]

3. Siedenbiedel, F.; Tiller, J.C. Antimicrobial polymers in solution and on surfaces: Overview and functional principles. Polymers 2012, 4, 46-71. [CrossRef]

4. Liu, X.; Zhang, H.; Tian, Z.; Sen, A.; Allcock, H.R. Preparation of quaternized organic-inorganic hybrid brush polyphosphazene-co-poly[2-(dimethylamino)ethyl methacrylate]electrospun fibers and their antibacterial properties. Polym. Chem. 2012, 3, 2082-2091. [CrossRef]

5. Willyard, C. Drug-resistant bacteria ranked. Nature 2017, 543, 15. [CrossRef] [PubMed]

6. Klevens, R.M.; Morrison, M.A.; Nadle, J.; Petit, S.; Gershman, K.; Ray, S.; Harrison, L.H.; Lynfield, R.; Dumyati, G.; Townes, J.M.; et al. Invasive methicillin-resistant Staphylococcus aureus infections in the United States. J. Am. Med. Assoc. 2007, 298, 1763-1771. [CrossRef] [PubMed]

7. Su, Y.; Tian, L.; Yu, M.; Gao, Q.; Wang, D.; Xi, Y.; Yang, P.; Lei, B.; Ma, P.X.; Li, P. Cationic peptidopolysaccharides synthesized by 'click' chemistry with enhanced broad-spectrum antimicrobial activities. Polym. Chem. 2017, 8, 3788-3800. [CrossRef]

8. Gao, Q.; Li, P.; Zhao, H.; Chen, Y.; Jiang, L.; Ma, P.X. Methacrylate-ended polypeptides and polypeptoids for antimicrobial and antifouling coatings. Polym. Chem. 2017, 8, 6386-6397. [CrossRef]

9. Allen, M.J.; White, G.F.; Morby, A.P. The response of Escherichia coli to exposure to the biocide polyhexamethylene biguanide. Microbiology 2006, 152, 989-1000. [CrossRef] [PubMed]

10. Chindera, K.; Mahato, M.; Sharma, A.K.; Horsley, H.; Kloc-Muniak, K.; Kamaruzzaman, N.F.; Kumar, S.; McFarlane, A.; Stach, J.; Bentin, T.; et al. The antimicrobial polymer PHMB enters cells and selectively condenses bacterial chromosomes. Sci. Rep. 2016, 6, 23121. [CrossRef] [PubMed]

11. Zhao, Y.; Lord, M.S.; Stenzel, M.H. A polyion complex micelle with heparin for growth factor delivery and uptake into cells. J. Mater. Chem. B 2013, 1, 1635-1643. [CrossRef]

12. Hemp, S.T.; Smith, A.E.; Bunyard, W.C.; Rubinstein, M.H.; Long, T.E. RAFT polymerization of temperatureand salt-responsive block copolymers as reversible hydrogels. Polymer 2014, 55, 2325-2331. [CrossRef] [PubMed]

13. Cao, X.; An, Z. RAFT synthesis in water of cationic polyelectrolytes with tunable UCST. Macromol. Rapid Commun. 2015, 36, 2107-2110. [CrossRef] [PubMed]

14. Lutz, J.-F.; Lehn, J.-M.; Meijer, E.W.; Matyjaszewski, K. From precision polymers to complex materials and systems. Nat. Rev. Mater. 2016, 1, 16024. [CrossRef] 
15. Stuart, M.A.C.; Huck, W.T.S.; Genzer, J.; Müller, M.; Ober, C.; Stamm, M.; Sukhorukov, G.B.; Szleifer, I.; Tsukruk, V.V.; Urban, M.; et al. Emerging applications of stimuli-responsive polymer materials. Nat. Mater. 2010, 9, 101-113. [CrossRef] [PubMed]

16. Stopiglia, C.D.O.; Collares, F.M.; Ogliari, F.A.; Piva, E.; Fortes, C.B.B.; Samuel, S.M.W.; Scroferneker, M.L. Antimicrobial activity of [2-(methacryloyloxy)ethyl]trimethylammonium chloride against Candida spp. Rev. Iberoam. Micol. 2012, 29, 20-23. [CrossRef] [PubMed]

17. Prijck, K.D.; Smet, N.D.; Coenye, T.; Schacht, E.; Nelis, H.J. Prevention of Candida albicans biofilm formation by covalently bound dimethylaminoethylmethacrylate and polyethylenimine. Mycopathologia 2010, 170, 213-221. [CrossRef] [PubMed]

18. Goel, N.K.; Rao, M.S.; Kumar, V.; Bhardwaj, Y.K.; Chaudhari, C.V.; Dubey, K.A.; Sabharwal, S. Synthesis of antibacterial cotton fabric by radiation-induced grafting of [2-(methacryloyloxy)ethyl]trimethylammonium chloride (MAETC) onto cotton. Radiat. Phys. Chem. 2009, 78, 399-406. [CrossRef]

19. Hassan, M.M. Binding of a quaternary ammonium polymer-grafted-chitosan onto a chemically modified wool fabric surface: Assessment of mechanical, antibacterial and antifungal properties. RSC Adv. 2015, 5, 35497-35505. [CrossRef]

20. Yanisch-Perron, C.; Vieira, J.; Messing, J. Improved M13 phage cloning vectors and host strains: Nucleotide sequences of the M13mp18 and pUC19 vectors. Gene 1985, 33, 103-111. [CrossRef]

21. Sambrook, J.; Russell, D.W. Molecular Cloning: A Laboratory Manual, 3rd ed.; Cold Spring Harbor Laboratory Press: Huntington, NY, USA, 2001.

22. Rawlinson, L.-A.B.; O'Gara, J.P.; Jones, D.S.; Brayden, D.J. Resistance of Staphylococcus aureus to the cationic antimicrobial agent poly(2-(dimethylamino ethyl)methacrylate) (pDMAEMA) is influenced by cell-surface charge and hydrophobicity. J. Med. Microbiol. 2011, 60, 968-976. [CrossRef] [PubMed]

23. Shirbin, S.J.; Lam, S.J.; Chan, N.J.-A.; Ozmen, M.M.; Fu, Q.; O’Brien-Simpson, N.; Reynolds, E.C.; Qiao, G.G. Polypeptide-based macroporous cryogels with inherent antimicrobial properties: The importance of a macroporous structure. ACS Macro Lett. 2016, 5, 552-557. [CrossRef]

24. Pranantyo, D.; Xu, L.Q.; Hou, Z.; Kang, E.-T.; Chan-Park, M.B. Increasing bacterial affinity and cytocompatibility with four-arm star glycopolymers and antimicrobial $\alpha$-polylysine. Polym. Chem. 2017, 8, 3364-3373. [CrossRef]

25. Lin, W.-C.; Liou, S.-H.; Kotsuchibashi, Y. Development and characterisation of the imiquimod poly(2-(2-methoxyethoxy)ethyl methacrylate) hydrogel dressing for keloid therapy. Polymers 2017, 9, 579. [CrossRef]

26. Berlutti, F.; Rosso, F.; Bosso, P.; Giansanti, F.; Ajello, M.; Rosa, A.D.; Farina, E.; Antonini, G.; Valenti, P. Quantitative evaluation of bacteria adherent to polyelectrolyte HEMA-based hydrogels. J. Biomed. Mater. Res. A 2003, 67A, 18-25. [CrossRef] [PubMed]

27. Williams, M.; Penfold, N.J.W.; Lovett, J.R.; Warren, N.J.; Douglas, C.W.I.; Doroshenko, N.; Verstraete, P.; Smets, J.; Armes, S.P. Bespoke cationic nano-objects via RAFT aqueous dispersion polymerization. Polym. Chem. 2016, 7, 3864-3873. [CrossRef]

28. He, H.; Adzima, B.; Zhong, M.; Averick, S.; Koepsel, R.; Murata, H.; Russell, A.; Luebke, D.; Takahara, A.; Nulwala, H.; et al. Multifunctional photo-crosslinked polymeric ionic hydrogel films. Polym. Chem. 2014, 5, 2824-2835. [CrossRef]

29. Yang, W.J.; Tao, X.; Zhao, T.; Weng, L.; Kang, E.-T.; Wang, L. Antifouling and antibacterial hydrogel coatings with self-healing properties based on a dynamic disulfide exchange reaction. Polym. Chem. 2015, 6, 7027-7035. [CrossRef]

30. Banerjee, I.; Pangule, R.C.; Kane, R.S. Antifouling Coatings: Recent Developments in the Design of Surfaces That Prevent Fouling by Proteins, Bacteria, and Marine Organisms. Adv. Mater. 2011, 23, 690-718. [CrossRef] [PubMed]

31. Lowe, S.; O’Brien-Simpson, N.M.; Connal, L.A. Antibiofouling polymer interfaces: Poly(ethylene glycol) and other promising candidates. Polym. Chem. 2015, 6, 198-212. [CrossRef]

(C) 2018 by the authors. Licensee MDPI, Basel, Switzerland. This article is an open access article distributed under the terms and conditions of the Creative Commons Attribution (CC BY) license (http:/ / creativecommons.org/licenses/by/4.0/). 